FACTA UNIVERSITATIS

Series: Mechanical Engineering Vol. 16, N² 2, 2018, pp. 219 - 232

https://doi.org/10.22190/FUME180227021C

Original scientific paper

\title{
DETERMINATION OF THE WALL VARIABLES WITHIN \\ THE ZONAL MODEL OF RADIATION INSIDE A PULVERIZED COAL-FIRED FURNACE
}

\author{
UDC 536.3, 621.6
}

\section{Nenad Crnomarković, Srđan Belošević, Stevan Nemoda, Ivan Tomanović, Aleksandar Milićević}

University of Belgrade, Vinča Institute of Nuclear Sciences, Serbia

\begin{abstract}
Determination of the wall variables (wall emissivities, wall temperatures, and heat fluxes) when the zonal model of radiation is used in numerical simulations of processes inside a pulverized coal-fired furnaces is described. Two methods for determination of the wall variables, i.e., a repeated run of numerical simulation (RRNS) and a temporary correction of the total exchange areas (TCTEA) are compared. Investigation was carried out for three values of the flame total extinction coefficient and four values of the initial wall emissivities. Differences of the wall variables were determined using the arithmetic means (AMs) of the relative differences. The AMs of the relative differences of the wall variables increased with an increase in the flame total extinction coefficient and changed a little with an increase in the initial values of the wall emissivities. For the selected furnace, the smallest differences of the wall variables were obtained for $K_{t}=0.3 \mathrm{~m}^{-1}$ and $\boldsymbol{\varepsilon}_{w, i n}=0.7$. Although both methods can be used for determination of the wall variables, the RRNS method was recommended because the manipulation with files was easier for it.
\end{abstract}

Key words: Zonal model, Pulverized coal, Boiler furnace, Numerical simulation, Wall variables

Received February 27, 2018 / Accepted June 09, 2018

Corresponding author: Nenad Crnomarković

University of Belgrade, Vinča Institute of Nuclear Sciences, Mike Petrovića Alasa 12-14, Serbia

E-mail: ncrni@vin.bg.ac.rs 


\section{INTRODUCTION}

Pulverized-coal fired furnaces of the utility boilers are basically rectangular-shaped constructions, inside which complex processes of reactive turbulent two-phase flows with radiative heat exchange occur. Values of the thermo-fluid variables, such as velocity components, temperature, component concentrations, and others, in every point of furnaces are determined by numerical simulations. Except for thermo-fluid variables, the numerical simulations should reveal values of the wall variables, such as wall temperatures, wall emissivities, and heat fluxes. As the wall temperatures and wall emissivities are found on the basis of the heat fluxes, the wall variables determination is clearly a task of the radiation model. The objective of this investigation is determination of the wall variables when the zonal model is used as a radiation model in numerical simulations.

In numerical investigation of processes inside pulverized coal-fired furnaces, several radiation models are used: discrete ordinate model [1], discrete transfer model [2], spherical harmonics model [3], six-flux model [4], Monte Carlo [5], and zonal model [6]. Each of them except for the zonal model (and Monte Carlo, which is based on the same concept as the zonal one) easily takes into account changes of all wall variables during the calculation procedure of the numerical simulation. The zonal radiation model is characterized by a high level of accuracy, independent of radiative properties and other conditions of the radiative heat exchange calculation. The model is based on division of furnace space into volume zones and furnace walls into surface zones [7]. All zones are considered isothermal. Radiative heat exchange is found in interaction of every zone with all zones. The total extinction coefficient and scattering albedo are assigned to all volume zones to determine the direct exchange areas (DEAs) for every pair of zones. For the gray furnace walls, net radiative heat exchange is found using the total exchange areas (TEAs). The calculation procedure for the TEAs requires the values of the wall emissivities for all surface zones. One set of the TEAs is used in the case of gray medium. To take into account the non-gray behavior of the gas phase, the concept of the weighted sum of gray gases model, by which a real gas is replaced by a mixture of gray gases [7], is used. Parameters of that model, temperature dependent weighting coefficient and constant absorption coefficient of each gray gas, are used to calculate the directed flux areas, which are then used to find net radiative exchange of zones.

In the classical application of the zonal model, radiative properties of the medium (total extinction coefficient and scattering albedo) and boundary walls (wall emissivity) are not changed during the calculation procedure. In that or similar way, the zonal method was used for numerical investigations of industrial furnaces [8-10], to find incident radiative fluxes on the freeboard walls of a bubbling fluidized bed [11], and for numerical investigations of a pulverized coal-fired furnace [6,12]. Nonclassical applications of the zonal model were described for nonhomogeneous radiatively participating medium [13-16]. These methods were developed for the black-walled systems [13, 14, 16], or for nonscattering medium [15], while the walls of pulverized coal-fired furnaces, inside which is a scattering medium, are gray. Papers which describe determination of wall variables by numerical simulations when radiative heat exchange is solved by the zonal model are very rare. Crnomarkovic et al. [17] described the method of the zonal model application, here called the repeated run of numerical simulation (RRNS). The new method, here called the temporary correction of TEAs (TCTEA), is described in this paper. Values of the wall variables obtained by RRNS and TCTEA methods for various flame radiative properties and initial wall emissivities are 
determined and compared. The investigation is expected to provide the recommendation for one of the methods.

The investigation was carried out for the pulverized coal-fired furnace of the $210 \mathrm{MW}$ monoblock thermal unit, located in Obrenovac, Serbia. The furnace was fired by Kolubara lignite. Geometry of the furnace and coal properties were described [18]. In the following text, the mathematical model, the results of the investigation and conclusions are described.

\section{Mathematical Model of the Process and Methods OF THE ZONAL MODEL APPLICATION}

Mathematical model of the process inside the furnace describes a two-phase reacting flow with radiative heat exchange. Model was described in detail in $[6,18]$. Here, only the main characteristics are described.

The gas phase is described by the time averaged differential equations of conservation of the momentum, enthalpy, the concentrations of the gas-phase component, particle concentrations, turbulent kinetic energy, and the rate of turbulent kinetic energy dissipation, in Eulerian reference frame. The general form of the gas-phase equation is the following:

$$
\operatorname{div}(\rho \mathbf{U} \Phi)=\operatorname{div}\left(\Gamma_{\Phi} \operatorname{grad} \Phi\right)+S_{\Phi}+S_{\Phi, \mathrm{p}}
$$

where $\rho$ is gas-phase density $\left(\mathrm{kg} \mathrm{m}^{-3}\right), \mathbf{U}$ is gas-phase velocity $\left(\mathrm{m} \mathrm{s}^{-1}\right), \Phi$ is the variable of the gas phase, $\Gamma_{\Phi}$ is the transport coefficient for variable $\Phi\left(\mathrm{kg} \mathrm{m}^{-1} \mathrm{~s}^{-1}\right), S_{\Phi}$ is the source term, and $S_{\Phi, \mathrm{p}}$ is the source term due to the presence of particles. The set of equations was closed by an appropriate turbulence model [18], which connects the turbulent kinetic energy and rate of its dissipation. The differential equation for the pressure field is obtained from the combination of the continuity and momentum equations, by the SIMPLE algorithm.

The flame temperatures are solved from the enthalpy equation for the condition of the thermal equilibrium between the gas and dispersed phases [6]:

$$
\operatorname{div}\left(\rho \mathbf{U} c_{\mathrm{f}} T_{\mathrm{f}}\right)=\operatorname{div}\left(\Gamma_{H} \operatorname{grad}\left(c_{\mathrm{f}} T_{\mathrm{f}}\right)\right)+\mathbf{U g r a d} P+S_{H, \mathrm{r}}+S_{H, \mathrm{c}}
$$

where $c_{\mathrm{f}}$ is the specific heat capacity of flame $\left(\mathrm{J} \mathrm{kg}^{-1} \mathrm{~K}^{-1}\right), T_{\mathrm{f}}$ is flame temperature $(\mathrm{K}), H$ is enthalpy $\left(\mathrm{J} \mathrm{kg}^{-1}\right), P$ is pressure $\left(\mathrm{N} \mathrm{m}^{-2}\right), S_{H, \mathrm{r}}$ is the source term due to radiation $\left(\mathrm{W} \mathrm{m}^{-3}\right)$, and $S_{H, \mathrm{c}}$ is the source term due to combustion $\left(\mathrm{W} \mathrm{m}^{-3}\right)$.

The dispersed phase is described by the differential equations of motion and change of mass and energy in the Lagrangian reference frame. Motion of the particles is tracked along the trajectories with constant flow of particles. The particle velocity vector is the sum of the convective and the diffusion components. Heterogeneous reactions of the coal combustion are modeled in the kinetic-diffusion regime, as described in [19].

Radiative heat exchange is solved by the zonal model of radiation. The heat flux of a surface zone is determined from the following relation:

$$
q_{\mathrm{w}, i}=\frac{\sum_{m=1}^{M} \overline{G_{m} S_{i}} \boldsymbol{\sigma} T_{\mathrm{f}, m}^{4}+\sum_{n=1}^{N} \overline{S_{n} S_{i}} \boldsymbol{\sigma} T_{\mathrm{f}, n}^{4}-A_{i} \boldsymbol{\varepsilon}_{\mathrm{w}, i} \boldsymbol{\sigma} T_{\mathrm{w}, i}^{4}}{A_{i}}, \quad i=1, \ldots, N
$$


where $T_{\mathrm{w}}$ is wall temperature $(\mathrm{K}), \overline{G_{m} S_{i}}$ is volume-surface TEA $\left(\mathrm{m}^{2}\right), \overline{S_{n} S_{i}}$ is surfacesurface TEA $\left(\mathrm{m}^{2}\right), M$ is the total number of the volume zones, $N$ is the total number of the surface zones, $\boldsymbol{\sigma}$ is Stefan-Boltzmann constant $\left(\mathrm{W} \mathrm{K}^{-4} \mathrm{~m}^{-2}\right), \boldsymbol{\varepsilon}_{\mathrm{w}}$ is wall emissivity (-), and $A$ is the surface area $\left(\mathrm{m}^{2}\right)$. The sum of the first two terms in the numerator of formula (3) is the heat transfer rate of the gained energy whereas the third term is the heat transfer rate of the energy loss due to emission of radiation. The wall emissivity is a function of wall temperature: $\boldsymbol{\varepsilon}_{\mathrm{w}}=\boldsymbol{\varepsilon}_{\mathrm{w}}\left(T_{\mathrm{w}}\right)$.

The furnace wall is a composite wall, consisting of a metal wall layer and an ash deposit layer. One boundary surface of the furnace wall, i.e. the metal wall boundary surface, is in contact with the flow of steam-water mixture and the other boundary surface, i.e. boundary surface of the ash deposit layer, is in contact with the flame. The wall temperature and the wall emissivity are the properties of the ash deposit layer boundary surface in contact with the flame. The ash deposit layer is treated as a gray emitter of radiation [20], although there is evidence that does not support such an assumption [21]. The thickness of the metal wall is $4.0 \mathrm{~mm}$ whereas the thickness of the ash deposit layer is chosen according to the objectives of the investigations.

The temperature of metal wall boundary surface $T_{\mathrm{m}, \mathrm{sw}}(\mathrm{K})$ which is in contact with the steam-water mixture is determined on the basis of the convective heat transfer:

$$
T_{\mathrm{m}, \mathrm{ww}}=T_{\mathrm{sw}}+\frac{q_{\mathrm{w}, i}}{h}
$$

where $h=14.0 \mathrm{~kW} \mathrm{~m}^{-2} \mathrm{~K}^{-1}$ is the convection transfer coefficient [22] and $T_{\mathrm{sw}}=615.0 \mathrm{~K}$ is the temperature of the steam-water mixture. The temperature of metal wall boundary surface $T_{\mathrm{m}, \mathrm{a}}(\mathrm{K})$ which is in contact with the ash deposit layer is determined on the basis of the one-dimensional heat conduction:

$$
T_{\mathrm{m}, \mathrm{a}}=T_{\mathrm{m}, \mathrm{sw}}+q_{\mathrm{w}, i} \frac{l_{\mathrm{m}}}{k_{\mathrm{m}}}
$$

where $l_{\mathrm{m}}$ is metal wall thickness (m) and $k_{\mathrm{m}}$ is the thermal conductivity of metal wall (W $\mathrm{m}^{-1}$ $\left.\mathrm{K}^{-1}\right)$. The thermal conductivity of the metal wall depends on temperature $k_{\mathrm{m}}=k_{\mathrm{m}}\left(\tilde{T}_{\mathrm{m}}\right)$, where $\tilde{T}_{\mathrm{m}}(\mathrm{K})$ is the arithmetic mean $(\mathrm{AM})$ of metal wall boundary surface temperatures, $\tilde{T}_{\mathrm{m}}=\left(T_{\mathrm{m} . \mathrm{sw}}+T_{\mathrm{m}, \mathrm{a}}\right) / 2$. Wall temperatures $T_{\mathrm{w}}(\mathrm{K})$ are determined by formula (5) replacing $T_{\mathrm{m}, \mathrm{sw}}, \tilde{T}_{\mathrm{m}}, l_{\mathrm{m}}$, and $k_{\mathrm{m}}$, by $T_{\mathrm{m}, \mathrm{a}}, \tilde{T}_{\mathrm{a}}, l_{\mathrm{a}}$, and $k_{\mathrm{a}}$, respectively. Here, $\tilde{T}_{\mathrm{a}}$ is the AM of boundary surface temperatures (K), $l_{\mathrm{a}}$ is thickness $(\mathrm{m})$, and $k_{\mathrm{a}}$ is effective thermal conductivity (W $\left.\mathrm{m}^{-1} \mathrm{~K}^{-1}\right)$, all of the ash deposit layer. Dependences of $k_{\mathrm{a}}$ and $\boldsymbol{\varepsilon}_{w}$ on temperature are in the polynomial forms:

$$
\begin{aligned}
k_{\mathrm{a}} & =\sum_{j=0}^{7} a_{j}\left(\frac{\tilde{T}_{\mathrm{a}}}{1000}\right)^{j} \\
\boldsymbol{\varepsilon}_{\mathrm{w}} & =\sum_{j=0}^{7} b_{j}\left(\frac{T_{\mathrm{w}}}{1000}\right)^{j}
\end{aligned}
$$


using the diagrams given in [20]. Coefficients $a_{j}$ and $b_{j}$ can be found in [23]. Dependence of the metal wall thermal conductivity on temperature is that of carbon steel [24, 25].

Thermophysical properties of the gas phase are determined from the equation of the state, tabulated values and empirical relations. The set of equations is solved by the finite difference method. Discretization and linearization of the equations are achieved by the method of the control volumes and hybrid difference scheme. Stability of the iterative procedure is provided by the under-relaxation method [26].

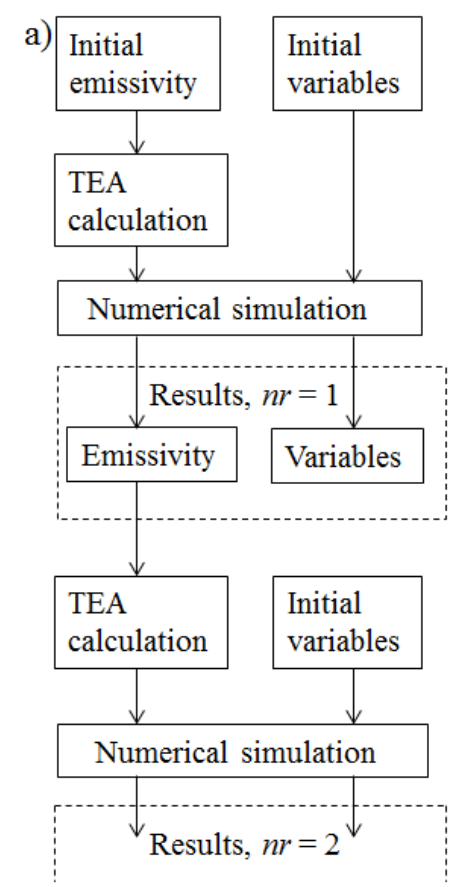

Fig. 1 Flow charts of the methods: a) RRNS, b) TCTEA

The flow charts of the RRNS and TCTEA methods are shown in Fig. 1a,b. The RRNS method starts with the determination of the TEAs using the initial wall emissivities and flame radiative properties. During the calculation procedure, wall temperatures $\left(T_{\mathrm{m}, \mathrm{sw}}\right.$, $T_{\mathrm{m}, \mathrm{a}}$, and $T_{\mathrm{w}}$ ) are determined on the basis of the heat fluxes and thermophysical properties of the wall. When the numerical simulation is completed, new values of the wall emissivities are determined in accordance with the wall temperatures, formula (6b). The TEAs are determined again and the numerical simulation is repeated with all variables (except wall emissivities) starting from their initial values, which are the same as in the previous numerical simulation. The TCTEA method is similar to the RRNS method. The main difference is in that the next run of the numerical simulation starts with the values of all variables obtained by the previous numerical simulation. 
The TCTEA method actually behaves as one numerical simulation. For the RRNS method, every new numerical simulation is independent of the previous one and contains the impact of the surface zone emissivities on the results. The necessary condition for applying the methods is the convergence, which must be shown using the variable values obtained by the numerical simulation.

\section{RESULTS AND DISCUSSION}

Radiative heat exchange was solved on the coarse numerical grid composed of the cubic volume zones of the edge dimension of $1.0 \mathrm{~m}$. The surface zones are squares of the same edge dimension. The furnace was divided into 7956 volume zones and 2712 surface zones. Flow field was solved on the fine numerical grid which was obtained by dividing every volume zone into 64 control volumes. The fine numerical grid contained 620136 control volumes. Agreement with experimental data and the grid independence study were already shown [6]. DEAs of the close zones were determined using correlations given in [10]. TEAs were calculated by the method of original emitters of radiation [7]. Improvements of the values of the TEAs were accomplished using the generalized Lawson's smoothing method [14]. One numerical simulation consisted of 4000 iterations.

The investigation was carried out for three values of the total extinction coefficient: (1) $K_{\mathrm{t}}=$ $0.3 \mathrm{~m}^{-1}$, (2) $K_{\mathrm{t}}=1.0 \mathrm{~m}^{-1}$, and (3) $K_{\mathrm{t}}=2.0 \mathrm{~m}^{-1}$, and for four values of the initial wall emissivities: $0.60,0.70,0.80$, and 0.90 . The scattering albedo was 0.5 for every value of $K_{\mathrm{t}}$. Such values of the total extinction coefficient were selected because the previous investigation [6] conducted for the same furnace showed that the heat transfer rates of the absorbed radiation (with constant wall emissivities and temperatures) and heat fluxes were maximal and almost constant for the values of $K_{\mathrm{t}}$ in the interval $0.2-2.0 \mathrm{~m}^{-1}$ and scattering albedo not bigger than 0.5 . The thickness of the ash deposit layer of $0.6 \mathrm{~mm}$ was uniform for all walls.

The wall variables were determined for surface zones. The analysis of the convergence of the methods included flame temperatures $T_{\mathrm{f}}$. The convergence of the methods was shown through the relative differences expressed by formula (7):

$$
\delta_{\eta, \text { cn }}=\frac{\left|\tilde{\eta}_{3}-\tilde{\eta}_{n r}\right|}{\tilde{\eta}_{3}} \cdot 100 \%
$$

where $n r$ designates the number of the numerical simulation run, $\eta$ designates the variable, and $\tilde{\eta}$ designates the AM of the variable:

$$
\tilde{\eta}=\sum_{i=1}^{N} \frac{\eta_{i}}{N}
$$

In formula (8), $N$ is the total number of surface zones that represent a solid wall (for $\boldsymbol{\varepsilon}_{\mathrm{w}}, T_{\mathrm{w}}$, and $q_{\mathrm{w}}$ ) or total number of control volumes (for $T_{\mathrm{f}}$ ). For the determination of the convergence, the numerical simulations were run eight times for every set of conditions. The similar values of the variables were obtained for both methods. The results obtained for $K_{\mathrm{t}}=1.0 \mathrm{~m}^{-1}$ are shown in Table 1 . For $n r \geq 3$, the changes of the AMs become very small and although the oscillations appear, both methods provide convergent results. The 
results obtained for the fourth run $(n r=4)$ of the numerical simulation were used for determination of the wall variable differences.

Table 1 Relative differences $\delta_{\eta, \text { cn }}(\%)$ obtained for $K_{\mathrm{t}}=1.0 \mathrm{~m}^{-1}, \boldsymbol{\varepsilon}_{\mathrm{w}, \text { in }}=0.80$

\begin{tabular}{lllllllll}
\hline \multirow{2}{*}{$N r$} & \multicolumn{2}{c}{$\boldsymbol{\varepsilon}_{\mathrm{W}}(-)$} & \multicolumn{2}{c}{$T_{\mathrm{w}}(\mathrm{K})$} & \multicolumn{2}{c}{$q_{\mathrm{w}}\left(\mathrm{kW} \mathrm{m}^{-2}\right)$} & \multicolumn{2}{c}{$T_{\mathrm{f}}(\mathrm{K})$} \\
\cline { 2 - 9 } & RRNS & TCTEA & RRNS & TCTEA & RRNS & TCTEA & RRNS & TCTEA \\
\hline 1 & 0.26 & 0.26 & 0.34 & 0.25 & 1.80 & 1.54 & 0.21 & 0.35 \\
2 & 0.0 & 0.0 & 0.003 & 0.002 & 0.009 & 0.22 & 0.003 & 0.22 \\
4 & 0.0 & 0.0 & 0.002 & 0.009 & 0.12 & 0.44 & 0.003 & 0.003 \\
5 & 0.0 & 0.0 & 0.004 & 0.001 & 0.23 & 0.007 & 0.001 & 0.10 \\
6 & 0.0 & 0.0 & 0.0009 & 0.004 & 0.001 & 0.14 & 0.002 & 0.009 \\
7 & 0.0 & 0.0 & 0.001 & 0.0002 & 0.004 & 0.0004 & 0.002 & 0.003 \\
8 & 0.0 & 0.0 & 0.0009 & 0.009 & 0.002 & 0.36 & 0.004 & 0.14 \\
\hline
\end{tabular}

In the following analyses, the changes and differences of the wall variables are determined through the AMs of the relative differences of the wall variables and the relative differences of the AMs of the heat fluxes, $\tilde{q}_{w}$. As the surface zones are squares of the same edge dimension, the AMs of the heat fluxes represent the heat transfer rates through the furnace walls.

The changes of the wall variable values with the change of the flame total extinction coefficient were found using the relative differences, determined by comparison of the wall variables with the values obtained for total extinction coefficient $K_{\mathrm{t}}=0.3 \mathrm{~m}^{-1}$ :

$$
\delta_{\eta, K_{\mathrm{t}}, i}=\frac{\left|\eta_{K_{\mathrm{t}}, i}-\eta_{0.30, i}\right|}{\eta_{0.30, i}} \cdot 100 \%
$$

The AMs of the relative differences of the wall variables and relative differences of the AMs of the heat fluxes are given in Table 2.

Table 2 AMs of relative differences $\boldsymbol{\delta}_{\eta, K_{\mathrm{t}}}(\%)$ of the wall variables and the relative differences of the AMs of the heat fluxes $\tilde{q}_{\mathrm{w}}(\%)$

\begin{tabular}{ccccccccccc}
\hline \multirow{2}{*}{$K_{\mathrm{t}}\left(\mathrm{m}^{-1}\right)$} & \multicolumn{4}{c}{$\eta$, RRNS } \\
\cline { 2 - 10 } & $\boldsymbol{\varepsilon}_{\mathrm{w}}$ & $T_{\mathrm{w}}$ & $q_{\mathrm{w}}$ & $\tilde{q}_{\mathrm{w}}$ & $q_{\mathrm{w}}{ }^{\mathrm{a}}$ & $\boldsymbol{\varepsilon}_{\mathrm{w}}$ & $T_{\mathrm{w}}$ & $q_{\mathrm{w}}$ & $\tilde{q}_{\mathrm{w}}$ & $q_{\mathrm{w}}{ }^{\mathrm{a}}$ \\
\hline 1.0 & 1.192 & 2.667 & 12.13 & 0.84 & 1.993 & 1.295 & 2.851 & 12.74 & 1.01 & 1.731 \\
2.0 & 1.777 & 3.828 & 17.04 & 0.55 & 1.425 & 1.991 & 4.183 & 18.36 & 0.50 & 0.328 \\
\hline \multicolumn{4}{c}{ a - By formula (9), without the absolute value sign }
\end{tabular}

The results show that the AMs of the relative differences of the wall variables increase with the increase in the total extinction coefficient of the flame. The values are similar for both methods. The biggest values of the AMs of the relative differences were obtained for heat fluxes. On the other hand, the relative differences of the AMs of the heat fluxes are much smaller. There are two reasons for such a difference. The first reason is in the use of an absolute-value sign which prevents the canceling out of positive and negative terms. 
The AMs of the relative differences of heat fluxes determined by formula (9) without the absolute value sign are shown in Table 2. They are much smaller than the AMs determined with the absolute-value sign. The second reason is in distribution of the relative differences along the furnace walls. The values of the heat fluxes determined by the TCTEA method for $K_{\mathrm{t}}$ $=0.3 \mathrm{~m}^{-1}$ and relative differences for $K_{\mathrm{t}}=1.0 \mathrm{~m}^{-1}$ and $K_{\mathrm{t}}=2.0 \mathrm{~m}^{-1}$ are shown in Fig. $2 \mathrm{a}$-c. It is evident that for some surface zones, the relative differences of the heat fluxes are big whereas the values of the heat fluxes are relatively small. Although such zones affect the relative differences of the AMs of the heat fluxes very little, they considerably influence the AMs of the relative differences of the heat fluxes.

The initial value of the surface zone emissivities must be determined in advance, as explained beforehand. The changes of wall variables with the change of the initial wall emissivity were found from the relative differences determined by comparison with the values obtained for the initial wall emissivity of 0.60 :

$$
\delta_{\eta, \varepsilon_{\text {w,in }}, i}=\frac{\left|\eta_{\varepsilon_{\mathrm{w}, \mathrm{in}}, i}-\eta_{0.60, i}\right|}{\eta_{0.60, i}} \cdot 100 \%
$$

The AMs of relative difference $\boldsymbol{\delta}_{\eta, \varepsilon_{w, i n}}$ are presented in Table 3 and show that the influence of the initial wall emissivity on the wall variables and their distribution along the furnace walls is very small. The wall variables are almost not affected by the initial wall emissivity if it is in the interval 0.60-0.90.

Table 3 AMs of relative differences $\delta_{\eta, \boldsymbol{\varepsilon}_{w, i n}}(\%), K_{\mathrm{t}}=0.3 \mathrm{~m}^{-1}$

\begin{tabular}{ccccccc}
\hline \multirow{2}{*}{$\boldsymbol{\varepsilon}_{\mathrm{w}, \text { in }}(-)$} & \multicolumn{3}{c}{$\eta$, RRNS } & \multicolumn{3}{c}{$\eta$, TCTEA } \\
\cline { 2 - 7 } & $\boldsymbol{\varepsilon}_{\mathrm{w}}$ & $T_{\mathrm{w}}$ & $q_{\mathrm{w}}$ & $\boldsymbol{\varepsilon}_{\mathrm{w}}$ & $T_{\mathrm{w}}$ & $q_{\mathrm{w}}$ \\
\hline 0.70 & 0.050 & 0.101 & 0.526 & 0.060 & 0.115 & 0.500 \\
0.80 & 0.042 & 0.084 & 0.434 & 0.108 & 0.201 & 0.909 \\
0.90 & 0.067 & 0.133 & 0.689 & 0.094 & 0.194 & 0.897 \\
\hline
\end{tabular}

The difference of the wall variable values caused by the selection of the method were found for three values of the total extinction coefficient: $K_{\mathrm{t}}=0.3,1.0$, and $2.0 \mathrm{~m}^{-1}$, and four initial wall emissivities: $0.6,0.7,0.8$, and 0.9 (only for $K_{\mathrm{t}}=0.3 \mathrm{~m}^{-1}$ ). The relative differences of the variables were determined by formula (11):

$$
\delta_{\eta, \mathrm{m}, i}=\frac{\left|\eta_{\mathrm{RRNS}, i}-\eta_{\mathrm{TCTEA}, i}\right|}{\eta_{\mathrm{TCTEA}, i}} \cdot 100 \%
$$



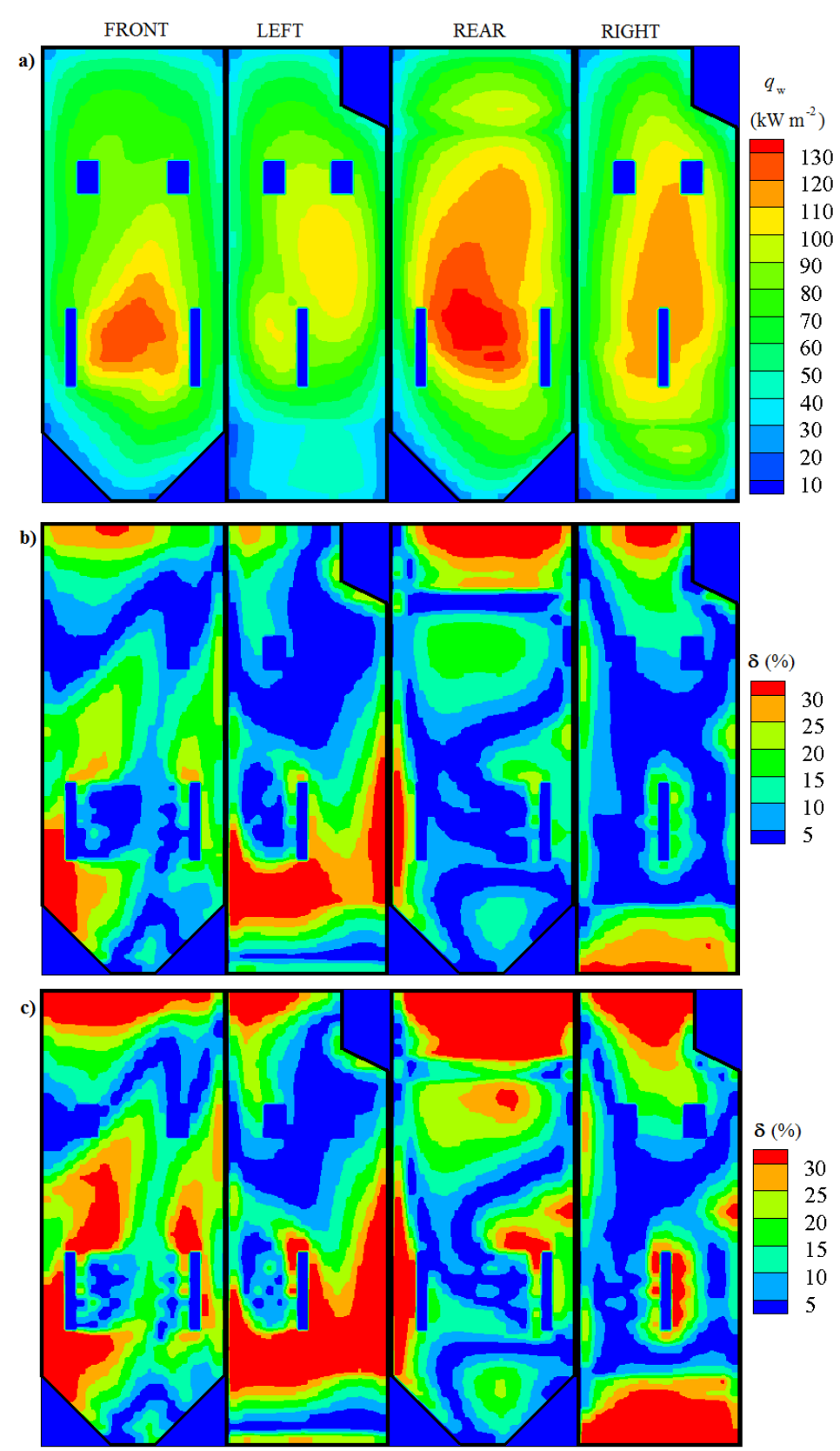

Fig. 2 Heat fluxes and relative differences, TCTEA method: (a) the heat fluxes for $K_{\mathrm{t}}=0.3 \mathrm{~m}^{-1}$, (b) relative differences $\boldsymbol{\delta}_{q_{\mathrm{w}}, K \mathrm{t}}$ for $K_{\mathrm{t}}=1.0 \mathrm{~m}^{-1}$, (c) relative differences $\boldsymbol{\delta}_{q_{\mathrm{w}}, K_{\mathrm{t}}}$ for $K_{\mathrm{t}}=2.0 \mathrm{~m}^{-1}$ 
The AMs of the relative differences are shown in Table 4. The AMs increase with an increase in the total extinction coefficient, and so the smallest AMs of the relative differences were obtained for $K_{\mathrm{t}}=0.3 \mathrm{~m}^{-1}$. The AMs of the relative differences of the wall variables for the selected initial wall emissivities are presented in the right half of Table 4 (for $\boldsymbol{\varepsilon}_{\mathrm{w}, \text { in }}=0.80$, the results are presented in the first column of the left half of Table 4). The AMs of the relative differences are almost constant for $\boldsymbol{\varepsilon}_{\mathrm{w} \text {,in }}$ from 0.6 to 0.80 , and the smallest values are obtained for $\boldsymbol{\varepsilon}_{\mathrm{w}, \text { in }}=0.70$. It is in the agreement with the previous result that the selection of the initial wall emissivities has small influence on the difference of the wall variables. The biggest AMs of the relative differences are obtained for the heat fluxes, for the same reason as previously. And again, the relative differences of the AMs of the heat fluxes are much smaller than the AMs of their relative differences.

Table 4 AMs of relative differences $\delta_{\eta, m}(\%)$ of the wall variables and the relative differences of the AMs of heat fluxes $\tilde{q}_{\mathrm{w}}(\%)$

\begin{tabular}{ccccccc}
\hline & \multicolumn{3}{c}{$K_{\mathrm{t}}\left(\mathrm{m}^{-1}\right)$} & \multicolumn{3}{c}{$\boldsymbol{\varepsilon}_{\mathrm{w}, \text { in }}(-)$} \\
\cline { 2 - 7 }$\eta$ & 0.3 & 1.0 & 2.0 & 0.60 & 0.70 & 0.90 \\
\hline $\boldsymbol{\varepsilon}_{\mathrm{w}}$ & 0.332 & 0.544 & 0.732 & 0.331 & 0.329 & 0.365 \\
$T_{\mathrm{w}}$ & 0.837 & 1.321 & 1.765 & 0.828 & 0.815 & 0.901 \\
$q_{\mathrm{w}}$ & 3.963 & 6.480 & 8.684 & 3.900 & 3.802 & 4.294 \\
$\tilde{q}_{\mathrm{w}}$ & 0.110 & 0.061 & 0.148 & 0.191 & 0.020 & 0.261 \\
\hline
\end{tabular}

The wall variable values determined by the RRNS method and for $K_{\mathrm{t}}=0.3 \mathrm{~m}^{-1}$ are shown in Fig. 3a-c. It is shown that the biggest wall temperatures and heat fluxes are obtained for the surface zones with the smallest emissivities. To further investigate their relation, the AMs of the wall emissivity, wall temperature, and heat fluxes were determined by the RRNS method for three thicknesses of the ash deposit layer. The results are shown in Table 5. It is clear that the increase of the ash deposit layer thickness reduces the heat exchange between the flame and furnace walls not only because it increases the wall temperatures but also because it reduces the wall emissivities. On the other hand, the fly-ash particles are the main contributor to the flame radiative properties. Without them, the radiative heat exchange inside the furnace would not be so intensive $[27,28]$.

Table 5 AMs of the wall emissivities, wall temperatures, and heat fluxes, $K_{\mathrm{t}}=0.3 \mathrm{~m}^{-1}$

\begin{tabular}{llcc}
\hline$l_{\mathrm{a}}(\mathrm{mm})$ & $\tilde{\boldsymbol{\varepsilon}}_{\mathrm{w}}(-)$ & $\tilde{T}_{\mathrm{w}}(\mathrm{K})$ & $\tilde{q}_{\mathrm{w}}\left(\mathrm{kW} \mathrm{m}^{-2}\right)$ \\
\hline 0.3 & 0.812 & 772.85 & 81.595 \\
0.6 & 0.777 & 870.20 & 74.379 \\
1.0 & 0.733 & 960.04 & 65.748 \\
\hline
\end{tabular}




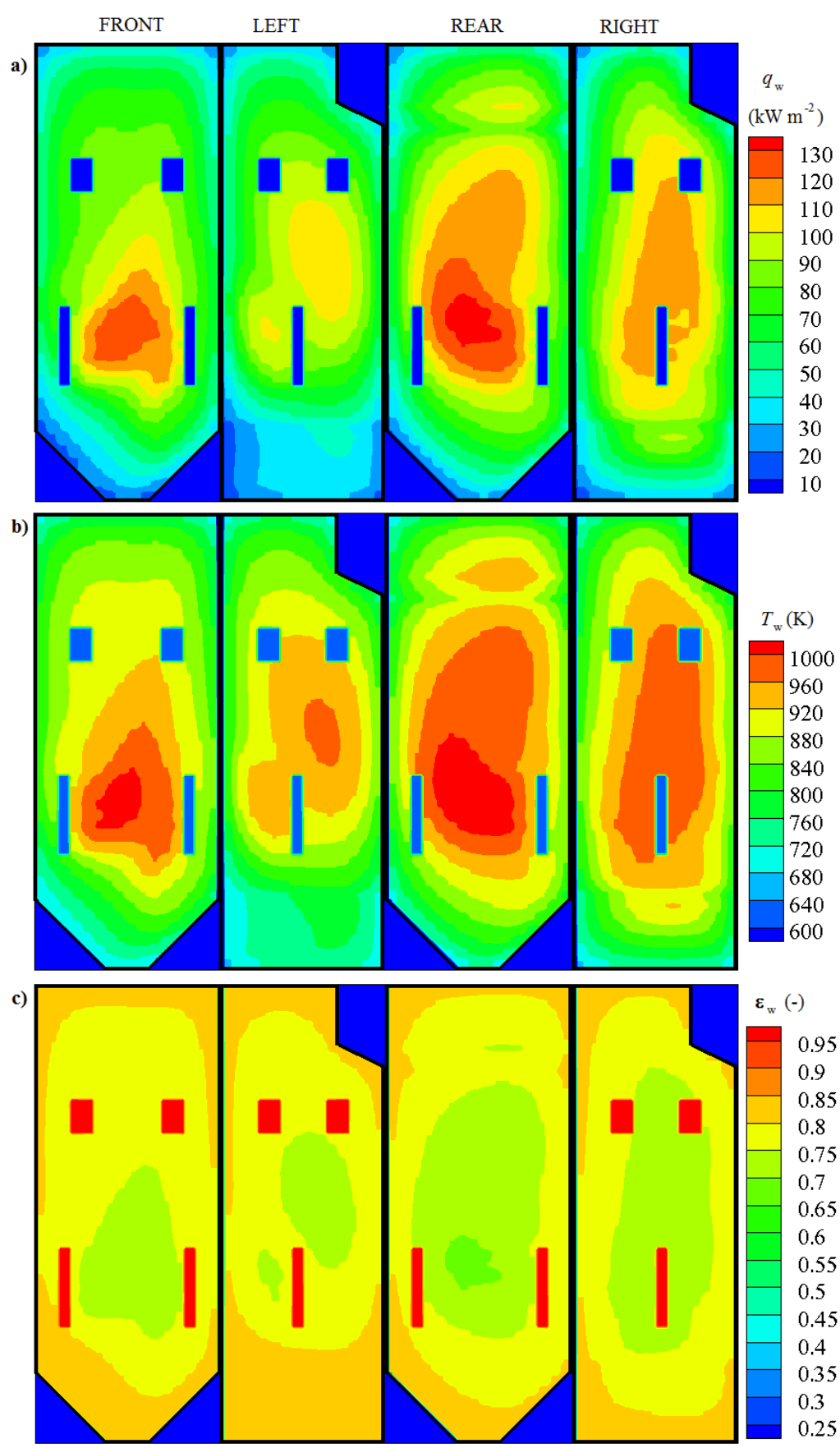

Fig. 3 Wall variables determined by the RRNS method, $K_{\mathrm{t}}=0.3 \mathrm{~m}^{-1}$ : (a) heat fluxes, (b) wall temperatures, (c) wall emissivities 
This investigation shows that any of the developed models, RRNS and TCTEA, can be used for determination of the wall variables. The methods are simple and can be easily applied. The RRNS method is recommended for the application in numerical simulations, because the manipulation with the files is easier for that method. The main drawback of the methods is the repetition of the numerical simulations, which is a consequence of the ash emissivity dependence on temperature. In the case of constant wall (that is ash layer) emissivity, the single run of numerical simulation would be enough to determine wall temperatures and heat fluxes. The objective of the further investigation is the formation of the new method by which the wall variables could be obtained from the single run of the numerical simulation. The main contribution of such investigation would be to improve the application of the zonal model in numerical simulations.

\section{CONCLUSIONS}

Two methods for determination of the wall variables: RRNS and TCTEA, for use in numerical simulations of processes inside pulverized coal-fired furnaces are compared. The radiative heat exchange is calculated using the zonal model of radiation. The changes and differences of the wall variables are determined for various conditions of the radiative heat exchange. The following conclusions are attained.

- For both methods, the AMs of the relative differences of the wall variables increase with an increase in the total extinction coefficient of the flame. The biggest increases of the AMs of the relative differences are obtained for the heat fluxes, whereas the relative differences of the AMs of the heat fluxes are much smaller. That is a consequence of the method of calculation and distribution of the relative differences along the furnace walls.

- The initial values of the wall emissivities influence the AMs of the relative differences of the wall variables very little, for the initial wall emissivities in the interval 0.60-0.90.

- The differences of the wall variables caused by the selection of the method increase with an increase in the flame radiative properties and only slightly depend on the wall emissivities. For the selected furnace, the smallest differences of the wall variables are obtained for $K_{\mathrm{t}}=0.3 \mathrm{~m}^{-1}$ and $\boldsymbol{\varepsilon}_{w, i n}=0.70$.

- As the difference of the wall variables obtained by both methods is small, the RRNS method is recommended for application in numerical simulations.

- The investigation showed that the increase of the ash deposit layer thickness reduces the heat fluxes by increasing the wall temperatures and reducing the wall emissivities.

Acknowledgement: This work is a result of the project "Increase in energy and ecology efficiency of processes in pulverized coal-fired furnace and optimization of utility steam boiler air preheater by using in-house developed software tools" (project No. TR-33018), supported by the Ministry of Education, Science and Technological Development of the Republic of Serbia. 


\section{REFERENCES}

1. Yin, C., 2013, Refined weighted sum of gray gases model for air-fuel combustion and its impacts, Energy \& Fuels, 27(10), pp. 6287-6294.

2. Fang, Q., Wang, H., Wei, Y., Lei, L., Duan, X., Zhou, H., 2010, Numerical simulations of slagging characteristics in a down-fired, pulverized-coal boiler furmace, Fuel Processing Technology, 91(1), pp. 88-96.

3. Liu, H., Liu, Y., Yi, G., Nie, L., Che, D., 2013, Effects of air staging conditions on the combustion and NOx emission characteristics in a $600 \mathrm{MW}$ wall fired utility boiler using lean coal, Energy \& Fuels, 27(10), pp. 5831-5840.

4. Belošević, S., Tomanović, I., Beljanski, V., Tucaković, D., Živanović, T., 2015, Numerical prediction of processes for clean and efficient combustion of pulverized coal in power plants, Applied Thermal Engineering, 74, pp. 120-110.

5. Fan, J., Qian, L., Ma, Y., Sun., P., Cen, K., 2001, Computational modeling of pulverized coal combustion processes in tangentially fired furnaces, Chemical Engineering Journal, 81(1-3), pp. 261-269.

6. Crnomarkovic, N., Sijercic, M., Belosevic, S., Tucakovic, D., Zivanovic, T., 2014, Radiative heat exchange inside the pulverized lignite fired furnace for the gray radiative properties with thermal equilibrium between phases, International Journal of Thermal Sciences, 85, pp. 21-28.

7. Hottel, H.C., Sarofim, A.F., 1967, Radiative transfer, McGraw-Hill Book Company, New York.

8. Tan, C.-K., Jenkins, J., Ward, J., Broughton, J., Heeley, A., 2013, Zone modelling of the thermal performancses of a large-scale bloom reheating furnace, Applied Thermal Engineering, 50(1), pp. 1111-1118.

9. Zhou, W., Qiu, T., 2015, Zone modelling of radiative heat transfer in industrial furnaces using adjusted Monte-Carlo integral method for direct exchange area calculation, Applied Thermal Engineering, 81, pp. 161-167.

10. Rhine, J.M., Tucker, R.J., 1991, Modelling of gas-fired furnaces and boilers, McGraw Hill, New York.

11. Selcuk, N., Batu, A., Ayranci, I., 2002, Performance of method of lines solution of discrete ordinates method in the freeboard of a bubbling fluidized bed combustor, Journal of Quantitative Spectroscopy \& Radiative Transfer, 73(2-5), pp. 503-516.

12. Chudnovsky, B., Karasina, E., Livshits, B., Talanker, A., 1999, Development and application of zonal combustion model for on-line furnace analysis of $575 \mathrm{MW}$ tangential coal firing boiler, Proc. Fifth International Conference on Technologies and Combustion for a Clean Environment, Lisbon, Vol. I, pp. 583-592.

13. Pieri, G., Sarofim, A. F., Hottel, H. C., 1973, Radiant heat transfer in enclosures: extension of HottelCohen zone method to allow for concentration gradients, Journal of the Institute of Fuel, 46(388), pp. 321-330.

14. Mechi, R., Farhat, H., Guedri, K., Halouani, K., Said, R., 2010, Extension of the zonal method to inhomogeneous non-gray semi-transparent medium, Energy, 35(1), pp. 1-15.

15. Modest, M. F., 2013, Radiative heat transfer, Academic Press, New York.

16. Yuen, W. W., 2006, The multiple absorption coefficient zonal method (maczm), an efficient computational approach for the analysis of radiative heat transfer in multidimen sional inhomogeneous nongray media, Numerical Heat Transfer, Part B, 49(2), pp. 89-103.

17. Crnomarkovic, N.D., Belosevic, S.V., Tomanovic, I.D., Milicevic, A.R., 2016, A new method of the zonal model of radiative heat exchange application by which the correction of the surface zone total emissivities is possible, Proc. International Conference Power Plants 2016, Zlatibor, pp. 1-10.

18. Crnomarkovic, N., Sijercic, M., Belosevic, S., Tucakovic, D., Zivanovic, T., 2012, Influence of forward scattering on prediction of temperature and radiation fields inside the pulverized coal furnace, Energy, 45(1), pp. 160-168.

19. Belosevic, S., Sijercic, M., Crnomarkovic, N., Stankovic, B., Tucakovic, D., 2009, Numerical prediction of pulverized coal flame in utility boiler furnaces, Energy \& Fuels, 23(11), pp. 5401-5412.

20. Boow, J., Goard, P.R.C., 1969, Fireside deposits and their effect on heat transfer in a pulverized-fuelfired boiler. Part III: The influence of the physical characteristics of the deposit on its radiant emittance and effective thermal conductance, Journal of the Institute of Fuel, 42(346), pp. 412-419.

21. Goetz, G.J., Nskala, N.Y., Borio, R.W., 1979, Development of method for determining emissivities and absorptivities of coal ash deposits, Journal of Engineering for Power, 101(4), pp. 607-614.

22. Wall, T.F., Lowe, A., Wibberley, L.J., Stewart, I.McC., 1979, Mineral matter in coal and the thermal performance of large boilers, Progress in Energy and Combustion Science, 5(1), pp. 1-29. 
23. Crnomarkovic, N.D., Sijercic, M. A., Belosevic, S. V., Tucakovic, D. R., Zivanovic, T. V., Tomanovic, I. D., Stojanovic, A.D., 2014, Numerical determination of the impact of the ash deposit on the furnace walls to the radiative heat exchange inside the pulverized coal fired furnace, Proc. International Conference Power Plants 2014, Zlatibor, pp. 1-12

24. Kaye, G.W.C., Laby, T.H., 1995, Tables of physical and chemical constants, Longman, London.

25. Singer, J.G., 1991, Combustion fossil power, Combustion Engineering, Connecticut.

26. Sijercic, M., 1998, Mathematical modeling of complex turbulent transport processes, Yugoslav Society of Thermal Engineers and Vinca Institute of Nuclear Sciences, Belgrade, (in Serbian).

27. Brkic, Lj., Zivanovic, T., Tucakovic, D., 2002, Thermal calculation of steam boilers, Faculty of Mechanical Engineering, Belgrade, (in Serbian).

28. Blokh, A.G., 1988, Heat transfer in steam boiler furnaces, Hemisphere, New York. 